\title{
The Effect of Analysis Conditions on the Fidelity of Atom Probe Data of Zirconium Alloys
}

Benjamin Jenkins ${ }^{1}$, Chris Grovenor ${ }^{2}$ and Michael Moody ${ }^{3}$

${ }^{1}$ University of Oxford, OXFORD, England, United Kingdom, ${ }^{2}$ Oxford University, OXFORD, England, United Kingdom, ${ }^{3}$ University of Oxford, Oxford, England, United Kingdom

Zirconium alloys are employed as the fuel cladding in light water nuclear reactors. However, due to deleterious changes in their properties during operation, the fuel rods must be regularly replaced [1]. This leads to a reduction in economic efficiency (due to reactor downtime) and an increase in nuclear waste (due to incomplete burn-up of $U$ fuel pellets). It is, therefore, desirable to design $\mathrm{Zr}$ alloys capable of operating for longer times in these extreme environments. The exact mechanisms by which $\mathrm{Zr}$ alloys undergo these changes are not yet fully understood. In order to gain a fundamental understanding of these phenomena, it is necessary to complement modelling studies with high-resolution characterisation. Atom probe tomography (APT) is a 3dimensional characterisation technique with a unique combination of high spatial and chemical resolution, and has been extensively used to study $\mathrm{Zr}$ alloys [2-4]. However, despite several papers demonstrating that APT analysis conditions have a large impact of the quality of the experimental data in other material systems [5], there exists limited literature on what the effects are on the quality of data on $\mathrm{Zr}$ alloys. In this presentation, data from a series of experiments on two commercial $\mathrm{Zr}$ alloys (Zircaloy-2 and ZIRLO) will be used to demonstrate the impact of APT analysis conditions on the resulting data quality. Analysis conditions including operating temperature, laser pulse energy, and voltage pulse fraction have been varied, and the advantages and drawbacks of the resulting data will be discussed. Preliminary data analysis has indicated that the use of laser pulsing can lead to significant surface migration of species, especially Sn, and that voltage pulsing mitigates this effect (Figure 1). Understanding the degree of surface migration throughout an experiment is important because it reduces the spatial resolution of the technique. Since certain elements are more susceptible to surface migration, associated biases will arise in any quantitative analyses that are later performed on the data. Indeed, clustering of Sn ions is visible only in voltage-pulsed experiments (Figure 2) on neutron-irradiated Zircaloy 2 alloys, which allows the relationship between $\mathrm{Sn}$ and other solvent species in $\mathrm{Zr}$-based alloys to be investigated more accurately using APT. This increase in accuracy poses an opportunity for APT to provide more detailed information to the modelling community, as they attempt to predict the evolution of the nanostructure during irradiation. However, the implementation of voltage-pulsing mode to study $\mathrm{Zr}$ alloys is not straight forward, with samples more likely to fracture during analysis before a reasonable amount of data has been collected. Balancing the amount of collected data against data fidelity is a compromise in many fields, and this talk will advise how best this balance can be met in the characterisation of both irradiated and nonirradiated $\mathrm{Zr}$ alloys using atom probe tomography. The research used UKAEA's Materials Research Facility, which has been funded by and is part of the UK's National Nuclear User Facility and Henry Royce Institute for Advanced Materials. 

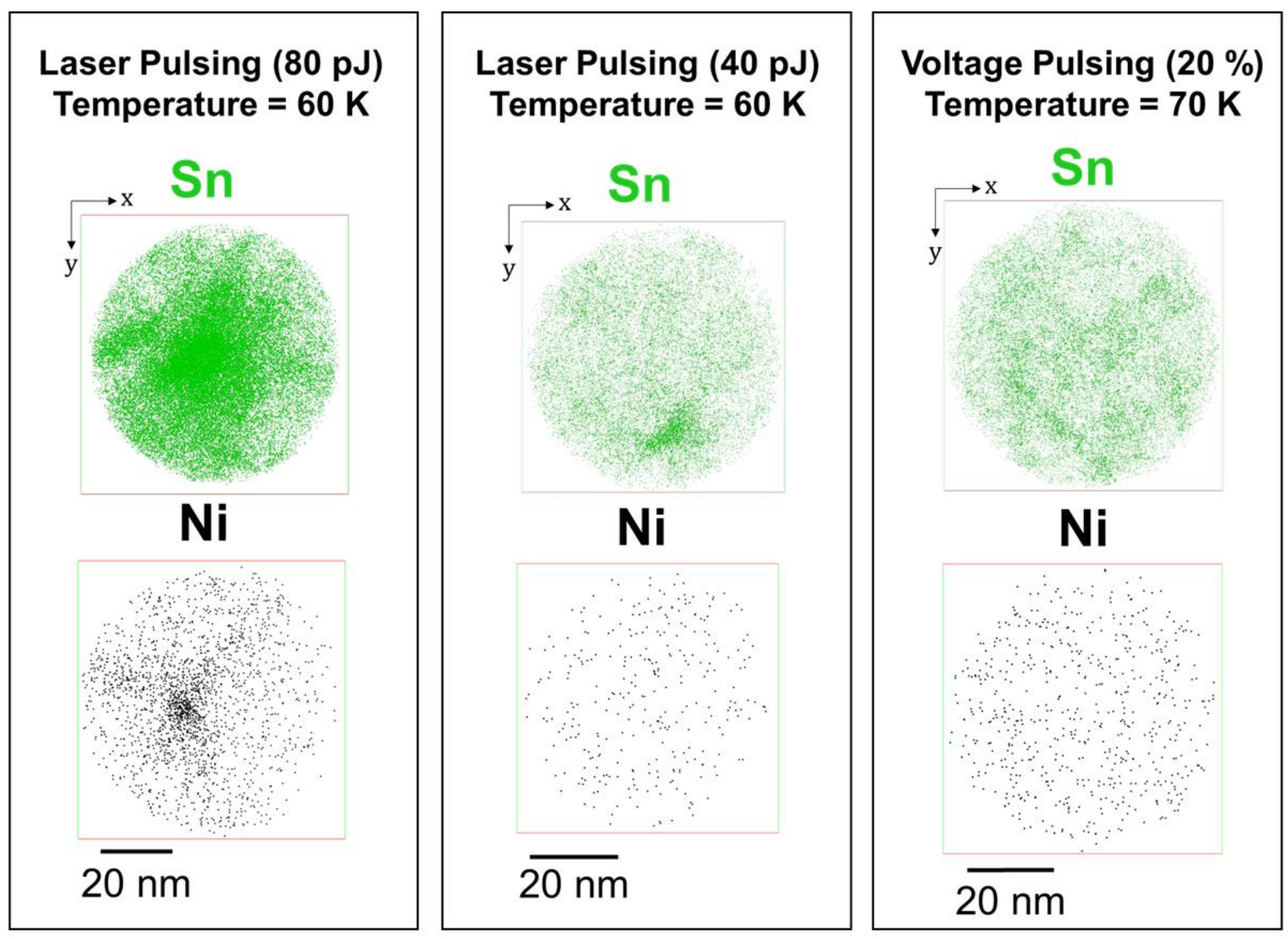

Figure 1. $\mathrm{x}-\mathrm{y}$ slices of atom maps demonstrating the reduction in surface migration of $\mathrm{Sn}$ and Ni when analysing a variety of $\mathrm{Zr}$ alloys with decreasing laser energy, and the use of voltage mode. 

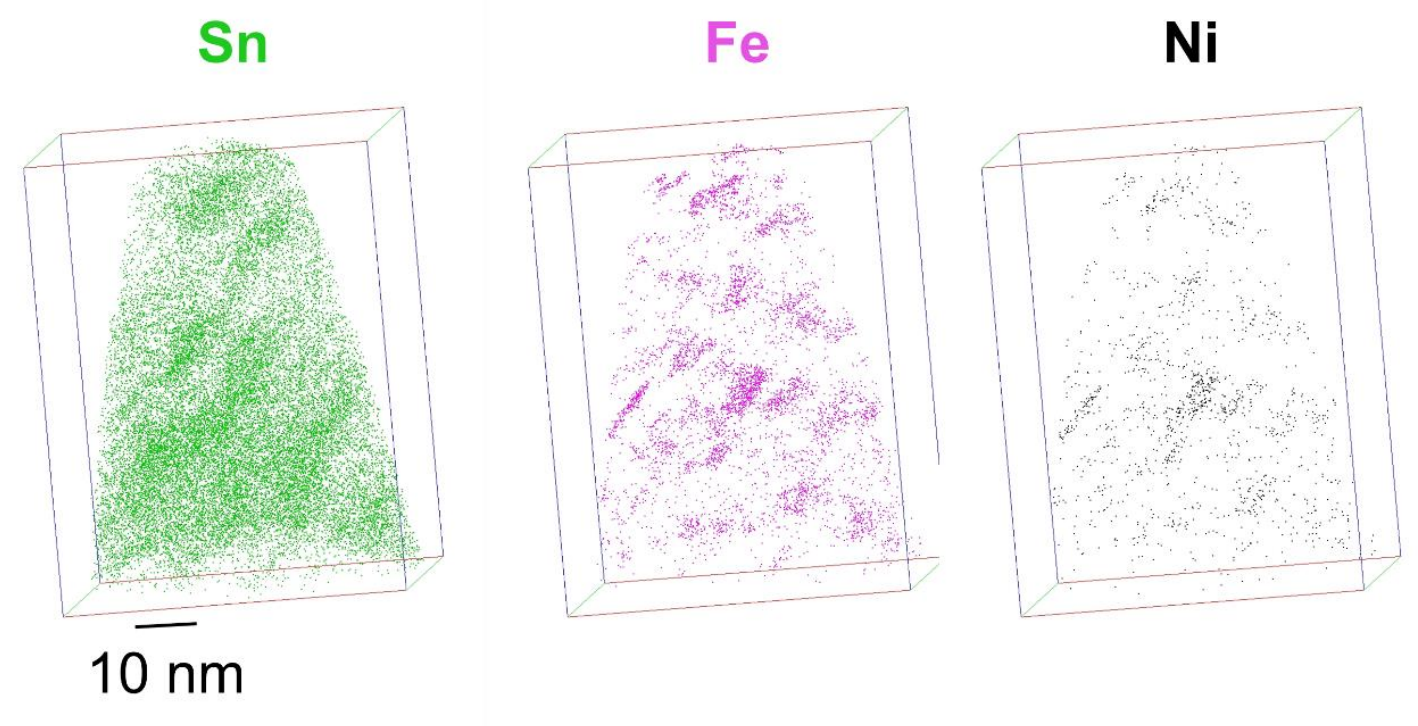

Figure 2. Atom maps showing the distribution of $\mathrm{Fe}, \mathrm{Sn}$, and $\mathrm{Ni}$ in a $\mathrm{Zr}-2$ alloy that was neutron-irradiated to $17 \mathrm{dpa}$. The experiment was conducted in voltage-pulsing mode at $70 \mathrm{~K}$. All three elements are observed to be non-randomly distributed in the dataset.

\section{References}

[1] S.J. Zinkle, K.A. Terrani, J.C. Gehin, L.J. Ott, L.L. Snead, Accident tolerant fuels for LWRs: A perspective, J. Nucl. Mater. 448 (2014) 374-379. doi:10.1016/j.jnucmat.2013.12.005.

[2] G. Sundell, M. Thuvander, H.O. Andrén, Tin clustering and precipitation in the oxide during autoclave corrosion of Zircaloy-2, J. Nucl. Mater. 456 (2015) 409-414. doi:10.1016/j.jnucmat.2014.10.003.

[3] G. Sundell, M. Thuvander, P. Tejland, M. Dahlbäck, L. Hallstadius, H.O. Andrén, Redistribution of alloying elements in Zircaloy-2 after in-reactor exposure, J. Nucl. Mater. 454 (2014) 178-185. doi:10.1016/j.jnucmat.2014.07.072.

[4] E. Francis, R. Prasath Babu, A. Harte, T.L. Martin, P. Frankel, D. Jädernäs, J. Romero, L. Hallstadius, P.A.J. Bagot, M.P. Moody, M. Preuss, Effect of $\mathrm{Nb}$ and $\mathrm{Fe}$ on damage evolution in a Zr-alloy during proton and neutron irradiation, Acta Mater. 165 (2019) 603-614. doi:10.1016/j.actamat.2018.12.021.

[5] J.M. Hyde, M.G. Burke, B. Gault, D.W. Saxey, P.D. Styman, K.B. Wilford, T.J. Williams, Atom probe tomography of reactor pressure vessel steels: an analysis of data integrity, Ultramicroscopy. 111 (2011) 676-682. doi:10.1016/j.ultramic.2010.12.033. 